\title{
Top-philic vectorlike portal to scalar dark matter
}

\author{
Stefano Colucci, ${ }^{1, *}$ Benjamin Fuks, ${ }^{2,3, \dagger}$ Federica Giacchino, ${ }^{4, \$}$ Laura Lopez Honorez, ${ }^{4,5,8}$ \\ Michel H. G. Tytgat, ${ }^{4, \|}$ and Jérôme Vandecasteele ${ }^{4, \pi}$ \\ ${ }^{1}$ Physikalisches Institut der Universität Bonn, Bethe Center for Theoretical Physics, \\ Nußallee 12, 53115 Bonn, Germany \\ ${ }^{2}$ Sorbonne Université, CNRS, Laboratoire de Physique Théorique et Hautes Énergies, \\ LPTHE, F-75005 Paris, France \\ ${ }^{3}$ Institut Universitaire de France, 103 boulevard Saint-Michel, 75005 Paris, France \\ ${ }^{4}$ Service de Physique Théorique, CP225, Université Libre de Bruxelles, \\ Bld du Triomphe, 1050 Brussels, Belgium \\ ${ }^{5}$ Vrije Universiteit Brussel and The International Solvay Institutes, Pleinlaan 2, 1050 Brussels, Belgium
}

(Received 20 April 2018; published 1 August 2018)

\begin{abstract}
We investigate the phenomenology of scalar singlet dark matter candidates that couple dominantly to the Standard Model via a Yukawa interaction with the top quark and a colored vectorlike fermion. We estimate the viability of this vectorlike portal scenario with respect to the most recent bounds from dark matter direct and indirect detection, as well as to dark matter and vectorlike mediator searches at colliders. Moreover, we take QCD radiative corrections into account in all our theoretical calculations. This work complements analyses related both to models featuring a scalar singlet coupled through a vectorlike portal to light quarks and to scenarios in which the dark matter is a Majorana singlet coupled to the Standard Model through scalar colored particles (akin to simplified models inspired by supersymmetry). Our study puts especially forward the complementarity of different search strategies from different contexts, and we show that current experiments allow for testing dark matter masses ranging up to $700 \mathrm{GeV}$ and mediator masses ranging up to $6 \mathrm{TeV}$.
\end{abstract}

DOI: 10.1103/PhysRevD.98.035002

\section{INTRODUCTION}

The hypothesis that dark matter (DM) may consist of weakly interacting massive particles (WIMPs) is currently being tested by various experiments including direct and indirect DM probes, as well as colliders searches. In this article, we study a minimal setup in which a real scalar DM particle $S$ couples to the Standard Model (SM) through interactions with a vectorlike fermion. Such a vectorlike portal scenario has been the object of several previous studies, which have focused on couplings either to light leptons [1-4] or to light quarks [5]. A distinctive feature of this class of portals is that radiative corrections tend to

\footnotetext{
*colucci@th.physik.uni-bonn.de

fuks@lpthe.jussieu.fr

federica.giacchino@ulb.ac.be

\$llopezho@ulb.ac.be

"mtytgat@ulb.ac.be

"jvdecast@ulb.ac.be
}

Published by the American Physical Society under the terms of the Creative Commons Attribution 4.0 International license. Further distribution of this work must maintain attribution to the author(s) and the published article's title, journal citation, and DOI. Funded by SCOAP ${ }^{3}$. play a major role in DM annihilation phenomenology. In particular, virtual bremsstrahlung or annihilations into monoenergetic photons and gluons may be the dominant mechanism driving the DM relic abundance. By the same token, these may give rise to remarkable spectral features, like a gamma-ray line that consists of a smoking gun for many DM searches. For physical and also technical reasons, previous studies have nevertheless been limited to couplings to light SM leptons or quarks. In this work, we complement these studies by considering a scenario in which the DM particle solely couples, at tree level, to the top quark through interactions with a vectorlike quark $T$. We explore different approaches to investigate the phenomenological viability of the model from both collider and cosmology standpoints.

In our predictions, we take into account several higherorder corrections that include the QCD Sommerfeld effect and next-to-leading-order (NLO) QCD corrections to the DM annihilation cross section, both in the early Universe and for what concerns indirect searches. For the latter purpose, we have in particular computed the $\mathcal{O}\left(\alpha_{s}\right)$ corrections to the $S S \rightarrow t \bar{t}$ annihilation process, which involves contributions from gluon emission by both the final-state top quarks and the virtual intermediate $t$-channel vectorlike mediator. Although the treatment of the associated 
infrared and collinear divergences is more involved for heavy quarks than when the DM candidate is coupled to light fermions, we only comment briefly on the associated difficulties and refer instead to Refs. [6,7] for details on the scalar DM and Majorana DM cases, respectively. We complement these constraints stemming from the relic density of DM and its indirect detection null results by a study of the relevance of existing direct DM probes. Our calculations take into account the effective coupling of the dark scalar $S$ to gluons through loops involving top quarks and $T$ mediators [8].

On different lines, we estimate how collider searches for both the vectorlike partner $T$ and the DM particle $S$ restrict the model. We extend a previous study relying on simplified model results from Run 1 of the LHC [9] by considering more recent LHC Run 2 supersymmetry searches that can be recast to constrain any model featuring strongly interacting quark partners decaying into a final state comprising missing energy and several SM objects [10]. We moreover include NLO QCD corrections through the computation of the corresponding matrix elements and match the fixed-order predictions with parton showers [11] so that a state-of-the-art modeling of the LHC signals is used. We additionally investigate the reach of the dedicated DM searches at the LHC in the mono-X channels in which the final-state signature consists of a pair of DM particles recoiling against a very hard SM object X.

The plan of this article is as follows. In Sec. II, we define the model and the associated parameter space. In Sec. III, we discuss our calculation of the DM relic abundance and how the latest results constrain the parameter space. In Sec. IV, we further derive bounds stemming from DM direct and indirect detection searches, and we finally address the collider phenomenology of the model in Sec. V. We emphasize the complementarity of the different approaches in Sec. VI, in which we summarize the various cosmological and collider bounds that we have obtained.

\section{THEORETICAL FRAMEWORK}

We consider a simplified top-philic DM setup in which we extend the Standard Model with a real scalar DM candidate $S$ with a mass $m_{S}$ and the interactions with the Standard Model of which are mediated by exchanges of a heavy vectorlike quark $T$ of mass $m_{T}$. The $T$ quark is as usual considered as lying in the fundamental representation of the QCD gauge group $S U(3)_{c}$, and we focus on a minimal option in which it is a weak isospin singlet with a hypercharge quantum number set to $2 / 3$. For the $S$ particle to be a stable DM candidate, we impose a $\mathbb{Z}_{2}$ discrete symmetry under which all Standard Model fields are even and the new physics states are odd. Provided the $\mathbb{Z}_{2}$ symmetry is unbroken, it prevents the $S$ field from mixing with the Standard Model Higgs doublet $\Phi$ and forbids the mixing of the $T$ quark with the Standard Model up-type quark sector.
Our model is described by the Lagrangian

$$
\begin{aligned}
\mathcal{L}= & \mathcal{L}_{\mathrm{SM}}+i \bar{T} \not D T-m_{T} \bar{T} T+\frac{1}{2} \partial_{\mu} S \partial^{\mu} S \\
& -\frac{1}{2} m_{S} S^{2}+\left[\tilde{y}_{t} S \bar{T} P_{R} t+\text { H.c. }\right]-\frac{1}{2} \lambda S^{2} \Phi^{\dagger} \Phi,
\end{aligned}
$$

where $P_{R}$ denotes the right-handed (RH) chirality projector and $t$ denotes the top-quark field. The interaction strength between the mediator $T$, the DM, and the SM sector (or equivalently the top quark) is denoted by $\tilde{y}_{t}$. Like the $\mathrm{DM}$, the vectorlike mediator field $T$ is odd under $\mathbb{Z}_{2}$ but otherwise transforms as the RH top field under $S U(3) \times$ $S U(2) \times U(1)$ (and so has electric charge $Q=+2 / 3$ ). A similar effective Lagrangian has been considered in Ref. [5] in the case of a DM particle coupling to light quarks and, more recently, in Refs. [12,13] for a coupling to the top quark. In contrast to these last two studies, our analysis differs in the treatment of the radiative corrections that are relevant for the relic abundance, DM indirect and direct detection, and for the modeling of the collider signals.

The core of this work focuses on the phenomenological implications of the presence of a colored vectorlike $T$ particle mediating the interactions of dark matter with the Standard Model. We therefore assume that the coupling of the DM particle to the Higgs boson $\lambda$ appearing in the Lagrangian of Eq. (2.1) can be neglected, so we set $\lambda=0$. We moreover impose that any loop contribution to $\lambda$ could be absorbed in the renormalization procedure and thus ignored. Details on departures from this hypothesis can be found in Ref. [12]. This contrasts with the case of a Majorana dark matter fermion that couples to the SM top quark through a scalar colored mediator. In the latter new physics setup, an effective DM-Higgs coupling arises at the one-loop level and it is calculable and finite [14].

The relevant model parameter space is therefore defined by three parameters, namely, the two new physics masses $m_{T}$ and $m_{S}$ and the Yukawa coupling $\tilde{y}_{t}$.

\section{DARK MATTER RELIC DENSITY}

\section{A. Radiative corrections}

It has been recently shown that radiative corrections to the DM annihilation cross section play a significant role in the phenomenology of a real scalar DM candidate, either through internal bremsstrahlung or via new channels that open up (like, for instance, when DM annihilates into a pair of monochromatic gluons or photons) [1-5]. All these analyses have, however, been restricted to scenarios featuring a DM particle coupling to light SM quarks or leptons, so the corresponding fermion masses could be neglected and the calculation could be performed in the so-called chiral limit. When nonvanishing SM fermion masses are accounted for, the computation of the radiative corrections to the annihilation cross section is plagued by infrared divergences that must be consistently handled, as has been 
studied in details for Majorana DM [15]. The scalar DM case has been thoroughly analyzed by some of us [6], so we summarize in this section the points that are the most relevant for our study.

The calculation of the annihilation cross section associated with the $S S \rightarrow t \bar{t}$ process at $\mathcal{O}\left(\alpha_{S}\right)$ involves contributions both from final-state radiation (FSR) and from virtual internal bremsstrahlung (VIB) diagrams. The corresponding amplitudes exhibit a specific dependence on the kinematics, which reflects in distinguishable features in the spectrum of radiated photons or gluons. In particular, VIB tends to yield a final-state energy spectrum that peaks at high energies $E_{\gamma, g} \lesssim m_{S}$. While FSR contributions also lead to the emission of a hard gluon or photon [16], the related spectral feature is less remarkable than in the VIB case, unless VIB is relatively suppressed. For a fixed DM mass $m_{S}$, the relative FSR and VIB weights are controlled by the mass of the vectorlike mediator $m_{T}$ and by the final-state quark mass (i.e., the mass of the top quark $m_{t}$ ). FSR turns out to be less important as $m_{t} / m_{S}$ decreases, since the contribution to the annihilation cross section is proportional to the leading-order (LO) result, which, in an $s$-wave configuration, is helicity suppressed. In the chiral limit, $m_{t} / m_{S} \rightarrow 0$, and FSR can thus be neglected. On the other hand, the VIB spectral features are controlled by the $m_{T} / m_{S}$ mass ratio, and the energy spectrum peaks toward $E_{\gamma, g} \sim m_{S}$ as $T$ and $S$ become more mass degenerate.

For generic particle masses, both FSR and VIB features are present and must be accounted for. This consequently requires a consistent handling of the infrared and collinear divergences of the FSR amplitude, which are only cancelled out after including the virtual contributions as guaranteed by the Kinoshita-Lee-Nauenberg theorem. The associated computations are facilitated when carried out in an effective approach (with a contact $S S t \bar{t}$ interaction) that is appropriate for the annihilation of nonrelativistic DM particles in the soft and collinear limit $[6,15]$. The hard part of the spectrum is then described by the $S S \rightarrow t \bar{t} g$ (or $t \bar{t} \gamma$ ) contribution as calculated from the full theory of Eq. (2.1), and the two results are matched by using a cutoff on the energy of the radiated gluon (photon). This approach allows us to get a regularized expression for the total $S S$ annihilation cross section at the NLO accuracy that is valid for a broad range of parameters [6].

The procedure outlined above vindicates the fact that for a large part of the parameter space one may rely on a simple approximation for the total annihilation cross section,

$\left.\sigma v_{t \bar{t}}\right|_{\mathrm{NLO}} \approx \begin{cases}\sigma v_{t \bar{t}} & m_{S}<300 \mathrm{GeV}, \\ \left.\sigma v_{t \bar{t} g}\right|_{m_{t}=0}+\sigma v_{t \bar{t}} & m_{S}>300 \mathrm{GeV} .\end{cases}$

In this expression, $\sigma v_{t \bar{t}}$ is the $s$-wave contribution to the LO annihilation cross section,

$$
\sigma v_{t \bar{t}}^{s-\text { wave }}=\frac{3 \tilde{y}_{t}^{4}}{4 \pi m_{S}^{3}} \frac{m_{t}^{2}\left(m_{S}^{2}-m_{t}^{2}\right)^{3 / 2}}{\left(m_{S}^{2}+m_{T}^{2}-m_{t}^{2}\right)^{2}},
$$

and $\left.\sigma v_{t \bar{t} g}\right|_{m_{t}=0}$ is the ( $s$-wave) annihilation cross section as obtained in the chiral limit and when a single gluon radiation is included. Its explicit form can be found in Refs. $[2,4,6]$. The difference with the exact result is only large for $m_{S} \simeq m_{t}$ and reaches at most $30 \%$ beyond (see Fig. 2 discussed in the framework of Sec. III B). When $m_{S} \rightarrow m_{t}$, the treatment used for the derivation of $\sigma v_{t \bar{t} g}$ breaks down due to threshold corrections that affect the production of a top-antitop system nearly at rest [17], an artifact that is visible in Fig. 2 in the region below $m_{S}$ of about $300 \mathrm{GeV}$. For $m_{S} \sim 300 \mathrm{GeV},\left.\sigma v_{t \bar{t}}\right|_{\mathrm{NLO}} \approx \sigma v_{t \bar{t}}$. Whereas the procedure allowing us to deal with threshold effects relevant for this mass configuration is in principle well known [18], its implementation goes beyond the scope of our work. Those effects not only concern a narrow range of parameters, but in the absence of toponium bound states, they are also expected to yield small and subleading corrections to the LO annihilation cross section [6]. For this reason, the LO annihilation cross section $\sigma v_{t \bar{t}}$ is used for scalar masses below about $300 \mathrm{GeV}$. For larger scalar masses, we additionally include the contribution of internal bremsstrahlung, calculated in the massless quark limit. Such an approximation provides a smooth transition to the mass regime in which gluon emission consists of the dominant contribution to the annihilation cross section, i.e., for $m_{S}$ of a few $\mathrm{TeV}$ [6].

\section{B. Relic abundance}

To determine the relic abundance of the dark $S$ particle, we consider the freeze-out mechanism for DM production in the early Universe and make use of the MicrOMEGAs code [19], which we have modified in order to accommodate some of the particularities of our model. These include dark matter annihilations into a $t W b$ three-body final state once one lies below the top threshold, the radiative corrections mentioned in Sec. III A, and Sommerfeld effects. The latter especially affect vectorlike fermion annihilation and dark matter coannihilation with a mediator, those corrections contributing to the relic abundance by at most $15 \%$ (see Appendix A). In addition, DM annihilations into a $t W b$ system play a non-negligible role for DM masses lying in the $\left[\left(m_{t}+m_{W}\right) / 2, m_{t}\right]$ mass window, and we have included these contributions by evaluating them numerically with CALCHEP [20]. Finally, we have added the loop-induced $S S \rightarrow g g$ and $S S \rightarrow \gamma \gamma$ processes in the computation of the DM annihilation cross section $[3,4]$. The annihilation into gluons is in particular significant for DM masses below the top threshold.

We present the results in Fig. 1, under the form of two two-dimensional projections of our three-dimensional parameter space. In the left panel of the figure, we show the region of the $\left(m_{S}, \tilde{y}_{t}\right)$ plane for which there exists a 

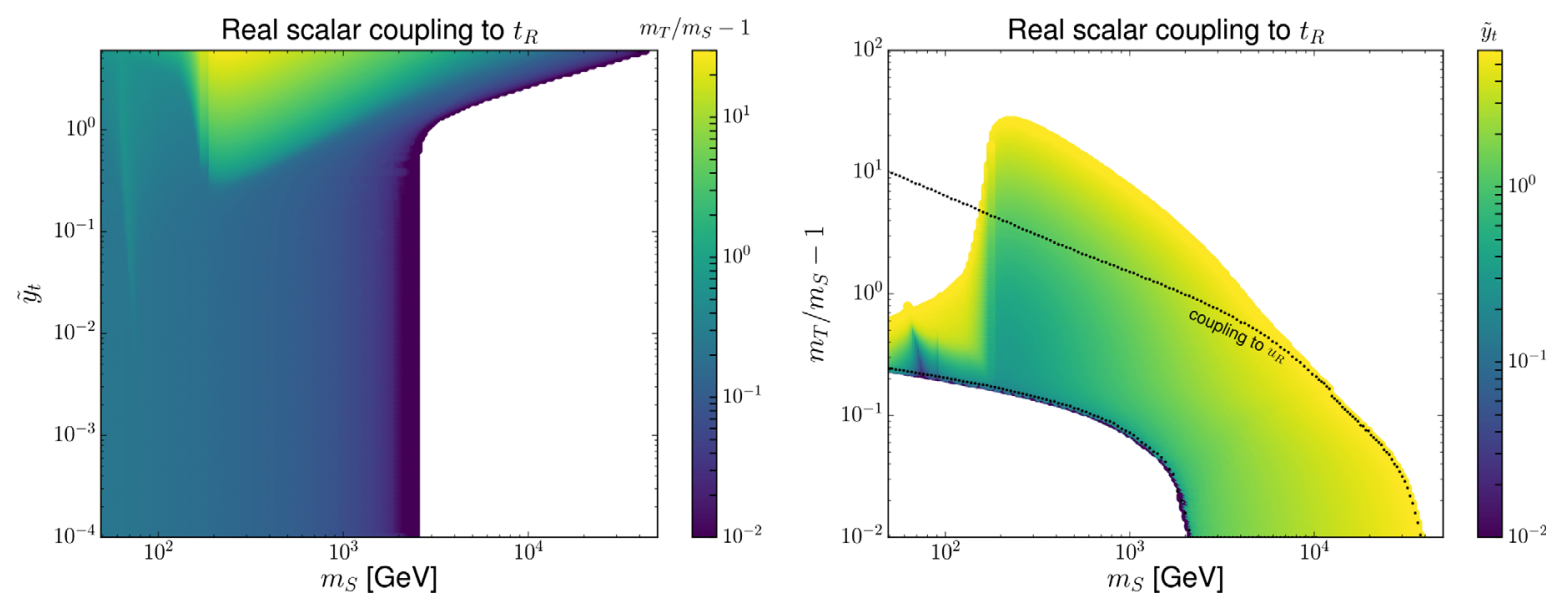

FIG. 1. Region of our parameter space for which one can accommodate a relic abundance of $\Omega h^{2}=0.12$. The results are shown in the $\left(m_{S}, \tilde{y}_{t}\right)$ plane (left) and $\left(m_{S}, r-1\right)$ plane (right), the color code being associated with the value of the $r-1$ and $\tilde{y}_{t}$ parameters, respectively. For comparison, the dotted black contour in the right panel represents the expected parameter space coverage in the case of a scalar DM particle coupling to right-handed up quarks $u_{R}$.

mediator mass value yielding to a relic density $\Omega h^{2}=0.12$ compatible with the Planck results [21]. The gradient of colors in Fig. 1 is associated to relative mass difference between the DM and the mediator given by $r-1$ with

$$
r=\frac{m_{T}}{m_{S}} .
$$

Similarly, we present in the right panel of the Fig. 1 the region of the $\left(m_{S}, r-1\right)$ plane for which there exists a $\tilde{y}_{t}$ coupling value, shown through a color code, leading to the observed relic abundance. The Yukawa coupling is enforced to lie in the $\left[10^{-4}, 6\right]$ window, the upper bound being an extreme value at the limit of the perturbative regime (defined by $\tilde{y}_{t} g_{s} / 4 \pi<1$ ) and the lower bound guaranteeing the correct treatment of the coannihilation processes by MicrOMEGAs. For $\tilde{y}_{t}>10^{-4}$, coannihilation processes like $S t \rightarrow T g$ occur in chemical equilibrium, and the DM abundance is determined by a single Boltzmann equation involving an effective annihilation cross section accounting for coannihilations [22]. For smaller $\tilde{y}_{t}$ values, thermal freeze-out could still yield the observed DM abundance, but a larger system of Boltzmann equations involving the abundance of both the $T$ and $S$ particles has to be accounted for in order to precisely determine the departure from chemical equilibrium [14,23]. This issue is left for a possible future work.

The two panels of Fig. 1 provide complementary information. In the $\left(m_{S}, \tilde{y}_{t}\right)$ plane, one observes parameter space regions in which the DM abundance is driven by coannihilation processes and so feature little dependence on the $\tilde{y}_{t}$ value. They correspond to setups for which $m_{T} / m_{S}-1$ is of at most $\mathcal{O}(0.1)$ and which are represented by the thin dark blue region in the complementary $\left(m_{S}\right.$, $\left.m_{T} / m_{S}-1\right)$ plane. For the sake of the comparison, we also superimpose in Fig. 1 the limits of the viable parameter space (black dotted contour) of a model for which the DM couples to the right-handed up quark $u_{R}$. We refer to Ref. [5] for more details.

The viable part of the parameter space can be divided into three distinct regions according to the DM mass $m_{S}$ :

(i) $m_{S}>5 \mathrm{TeV}$ : For very heavy DM, the mass of the top quark only plays a subleading role. This is clearly visible in the right panel of Fig. 1, in which the viable region of the parameter space of the topphilic scenario matches the one expected in the upphilic case. In this regime, $m_{S} \gg m_{t}$, and the chiral limit approximation for the DM annihilation cross section is valid. Moreover, VIB corrections are large, as illustrated in Fig. 2, in which we show, for all benchmark points giving rise to the right DM

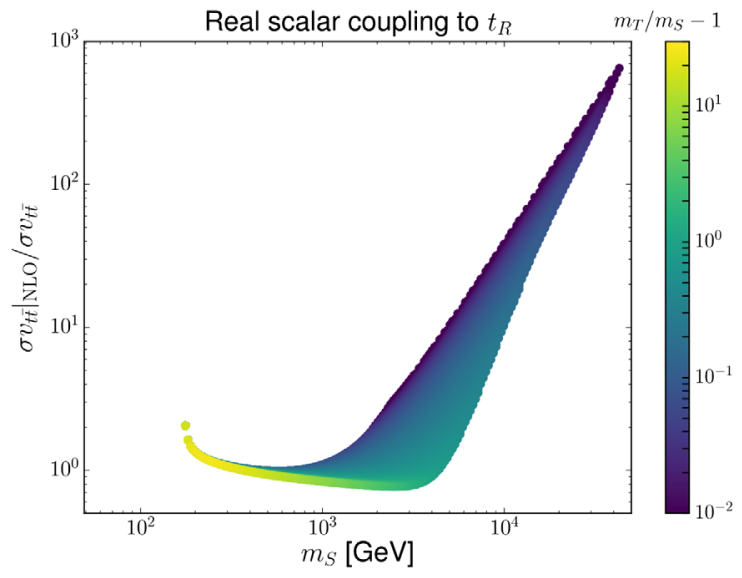

FIG. 2. Ratio of the exact NLO DM annihilation cross section $\left.\sigma v_{t \bar{t} g}\right|_{\mathrm{NLO}}[6]$ to the two-body LO cross section $\sigma v_{t \bar{t}}$. This shows that gluon radiation consists of the dominant component of the annihilation cross section for DM masses satisfying $m_{S} \gtrsim 5 \mathrm{TeV}$. In the figure, all points correspond to models matching the correct DM abundance, and the color code represents the value of $r-1$. 
abundance in Fig. 1, the ratio of the exact NLO result [6] to the LO predictions $\sigma v_{t \bar{t}}$. The importance of the NLO corrections will be further discussed in the context of DM indirect detection bounds in Sec. IV B.

(ii) $m_{t}<m_{S}<5 \mathrm{TeV}$ : In this regime in which the DM mass is moderate, the tree-level $s$-wave $S S \rightarrow t \bar{t}$ contribution to the annihilation cross section dominates, as additionally illustrated in Fig. 2, in which the NLO-to-LO ratio is close to 1 . Notice that the feature observed for $m_{S} \sim m_{t}$ in Fig. 2 is spurious as correct predictions must include threshold effects that we have ignored. The $\mathrm{LO}$ annihilation into a pair of quarks is, in contrast, completely negligible in the light quark case for which the relic density is driven by loop-induced annihilations into gluons [5]. The phenomenologically viable region of the parameter space in the top-philic scenario consequently strongly deviates from the corresponding one in the up-philic model, as shown in the right panel of Fig. 1. Given that finite quark mass effects are significant, larger $r$ parameters are found acceptable for a given DM mass in the top-philic case.

(iii) $m_{S}<m_{t}$ : In this regime, the DM abundance is driven either by annihilations into a $t W b$ system via a virtual top quark (for $m_{S} \lesssim m_{t}$ ), through loopinduced annihilations into gluons, or through coannihilations with the mediator. Any other potential contribution, like DM annihilations into pairs of SM particles through the Higgs portal (as it occurs in the scalar singlet DM scenario [24,25]), is irrelevant here since we have set the $\lambda$ quartic coupling in Eq. (2.1) to zero. Coannihilations particularly play an important role near $m_{T}+m_{S} \simeq m_{t}$, as the $S T \rightarrow t \rightarrow t g$ channel is resonantly enhanced. This corresponds to the light-yellow region in the left panel of Fig. 1 for $m_{S} \sim 70-80 \mathrm{GeV}$ and to the blue peak in the right panel of the figure for the same $m_{S}$ values. Annihilations into monochromatic gluons are only important when the mass of the mediator is large enough to close all coannihilation channels, and annihilations into a $t W b$ three-body system are only relevant close to threshold, for $m_{S} \in\left[\left(m_{t}+m_{W}\right) / 2, m_{t}\right]$.

\section{DIRECT AND INDIRECT CONSTRAINTS}

\section{A. Direct detection constraints}

In the limit in which the quartic coupling of $S$ to the Higgs boson vanishes, the DM nucleon scattering cross section can be computed from the evaluation of the oneloop diagrams shown in Fig. 3. This allows one to derive an effective Lagrangian for the DM coupling to gluons,

$$
\mathcal{L}_{g}=C_{S}^{g} \frac{\alpha_{s}}{\pi} S^{2} G^{\mu \nu} G_{\mu \nu}
$$
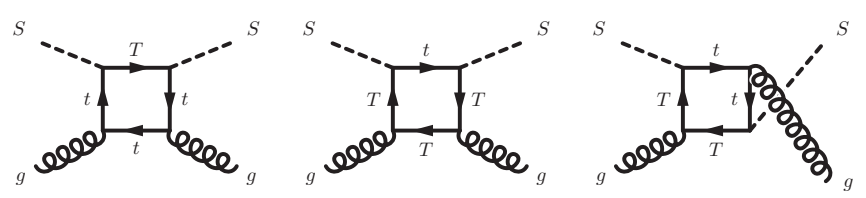

FIG. 3. Feynman diagrams relevant for DM-nucleon scattering.

where the Wilson coefficient $C_{S}^{g}$ includes both short- and long-distance contributions (relatively to the momentum scale involved in the loop) $[8,26]$. The resulting effective spin-independent coupling $f_{N}$ of the scalar DM particle $S$ to a nucleon $N$ of mass $m_{N}$ is then given by [27]

$\frac{f_{N}}{m_{N}}=-\frac{8}{9} C_{S}^{g} f_{T_{G}}^{(N)} \quad$ with $\quad f_{T_{G}}^{(N)}=1-\sum_{q=u, d, s} f_{T_{q}}^{(N)}$

where the quark mass fractions $f_{T_{q}}^{(N)}$ and the analytical expression for $C_{S}^{g}$ can be found in Ref. [28].

We compute the total spin-independent cross section $\sigma_{A}$ for DM scattering off a nucleus with charge $Z$ and a mass number $A$ by taking the coherent sum of the proton and the neutron contributions,

$$
\sigma_{A}=\frac{m_{A}^{2}}{\pi\left(m_{S}+m_{A}\right)^{2}}\left[Z f_{p}+(A-Z) f_{n}\right]^{2}
$$

where $f_{p}$ and $f_{n}$ denote the respective DM couplings to a proton and a neutron derived from Eq. (4.2) with $N=p$ or $n$, respectively, and $m_{A}$ is the nucleus mass.

In Fig. 4, we present the dependence of the DM scattering cross section on protons $\sigma_{S I}$ calculated as

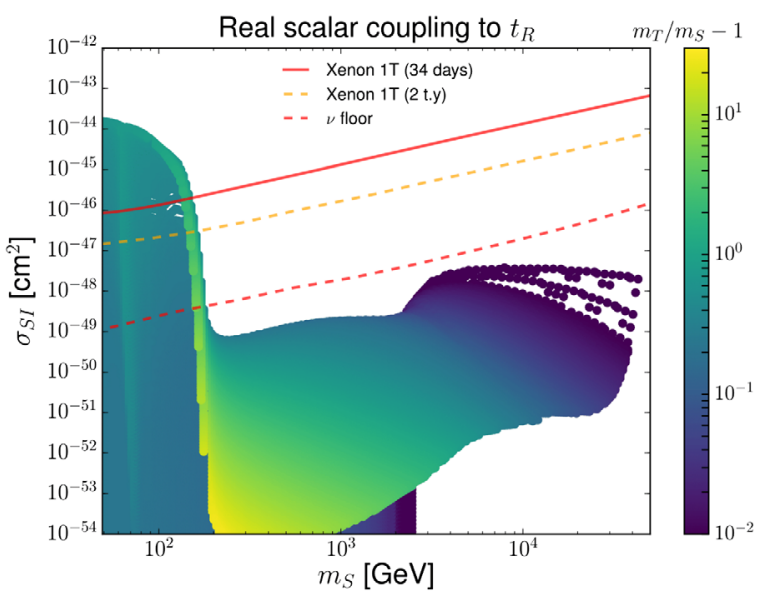

FIG. 4. DM-proton spin-independent scattering cross section as a function of the DM mass $m_{S}$. For each scenario, the coupling to the top quark and the value of the $r-1$ parameter (shown through the color code) are fixed to reproduce the observed relic density. The continuous red line represents the $90 \%$ confidence level exclusion of the Xenon 1T experiment [29], the orange dashed line represents the Xenon $1 \mathrm{~T}$ reach [30], and the red dashed line represents the neutrino floor [31]. 
depicted above, for all DM scenarios of Fig. 1. For $m_{S} \lesssim m_{t}$, the models featuring the largest $\sigma_{S I}$ values are those with the largest $\tilde{y}_{t}$ value and for which the relic density is driven by annihilations into a pair of gluons. As in the left panel of Fig. 1, the yellow region around $m_{S} \sim$ $80 \mathrm{GeV}$ corresponds to scenarios for which resonant coannihilations of the $S$ and $T$ particles into a top quark play a leading role. Above the top mass threshold, the Yukawa coupling required to match a correct relic abundance drops, and so does the elastic scattering cross section. The figure finally exhibits a bump above $m_{S} \gtrsim 2.5 \mathrm{TeV}$, which corresponds to setups in which $m_{S}+m_{t} \sim m_{T}$. The $C_{S}^{g}$ coefficient is then consequently enhanced, which directly impacts the elastic cross section [28].

For most DM models, $\sigma_{S I}$ lies, however, below the neutrino floor, except for some scenarios with a DM candidate lighter than the top quark. The constraints originating from the results of the Xenon 1T experiment after 34 days of exposure [29] are also indicated, together with predictions under the assumption of 2.1 years of data acquisition [30]. Although a large part of the parameter space region lying above the neutrino floor is within the range of Xenon 1T, a significant fraction of it will stay unconstrained in the near future by DM direct detection searches. The corresponding excluded region projected in the $\left(m_{S}, r-1\right)$ plane is presented in the summary of Fig. 11, after accounting for the latest bounds from the Xenon 1T experiment (red region), together with the region that could be tested up to the neutrino floor (red dashed contour).

\section{B. Indirect detection constraints}

In Figs. 5 and 6, we present, for all scenarios satisfying the relic density constraints of Sec. III, the value of the DM annihilation cross section at zero velocity into varied final states and using different approximations. In the upper panel of Fig. 5, we show the LO contribution to the $S S \rightarrow t \bar{t}$ channel, while the NLO corrections, computed in the approximation of Eq. (3.1), are included in the central panel. In the lower panel of the figure, we only show the gluon emission contributions, $S S \rightarrow t \bar{t} g$, computed in the chiral limit for $m_{S}>300 \mathrm{TeV}$. The (loop-induced) contributions of the $S S \rightarrow g g$ channel to the annihilation cross section are evaluated in Fig. 6.

Comparing the upper and central panels of Fig. 5, we observe that QCD emissions play a significant role for $m_{S}>2 \mathrm{TeV}$, as already visible in Fig. 2 (in which the exact NLO results from Ref. [6] have been employed). In contrast, Fig. 6 shows that annihilations into pairs of gluons are only relevant for $m_{S}<m_{t}$ (see also Sec. III). Moreover, $\sigma v_{g g}$ exhibits a minimum around $m_{S} \sim 280 \mathrm{GeV}$ independently of the value of $r$. This minimum is connected to a change of sign at the level of the loop amplitude that always happens for $m_{S} \in[270,290] \mathrm{GeV}$ (see Appendix B for an analytic expression of $\sigma v_{g g}$ ). As in Fig. 1, the yellow region around $m_{S} \sim 60-70 \mathrm{GeV}$ in Fig. 6 corresponds to
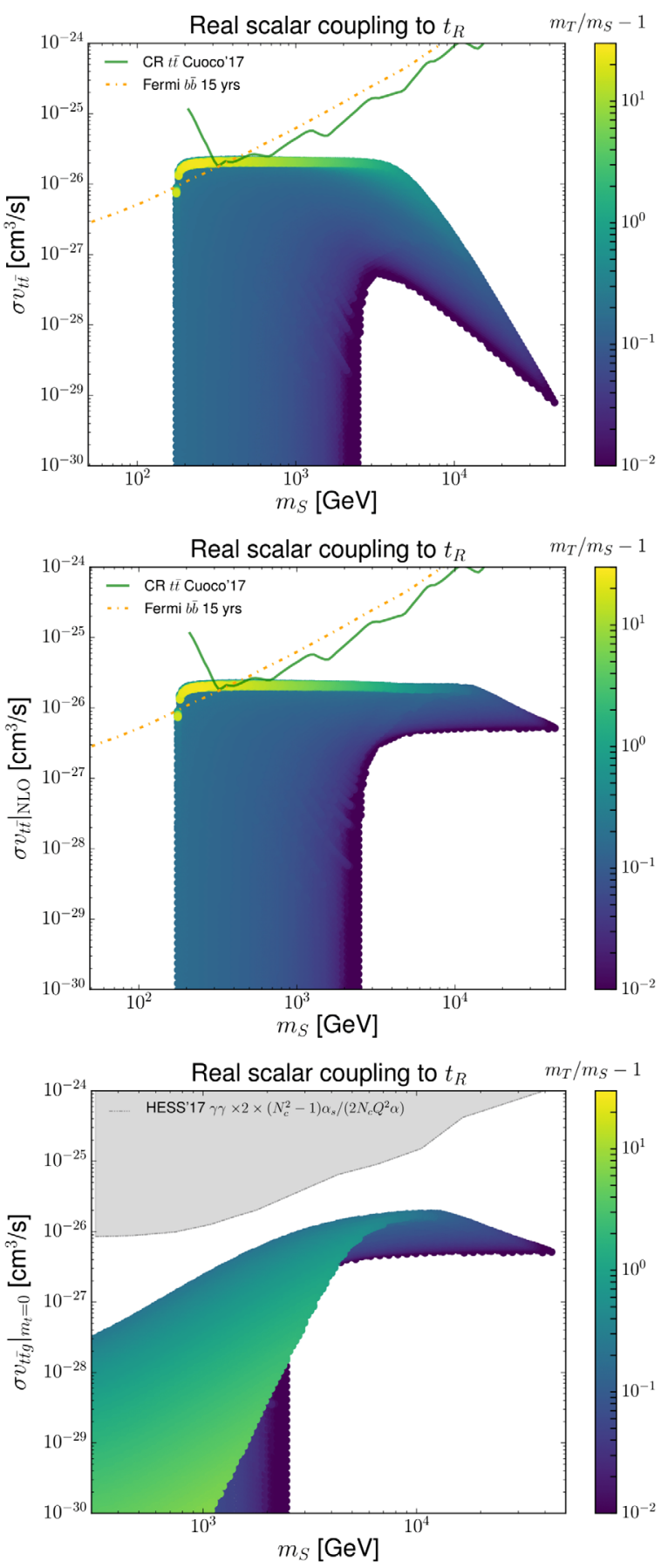

FIG. 5. LO (upper panel) and NLO (central panel) $S S \rightarrow t \bar{t}$ annihilation cross sections at zero velocity, where the NLO results are evaluated using the approximation of Eq. (3.1), as well as the $S S \rightarrow \bar{t} t g$ annihilation cross section in the chiral limit [2] (lower panel). We superimpose to our results the indirect detection limits obtained from the cosmic-ray (CR) analysis of Ref. [32] (green continuous line), as well as the bounds that could be expected after 15 years of Fermi-LAT running when dwarf spheroidal galaxy data in the $b \bar{b}$ channel are analyzed [33] (dot-dashed orange line). An estimation of the upper limits expected from gamma-ray line H.E.S.S. data [34] is also presented (see the text for details) in the lower panel (gray). The color code represents the value of the $r-1$ parameter, and we have considered DM models satisfying the relic density constraints of Sec. III. 


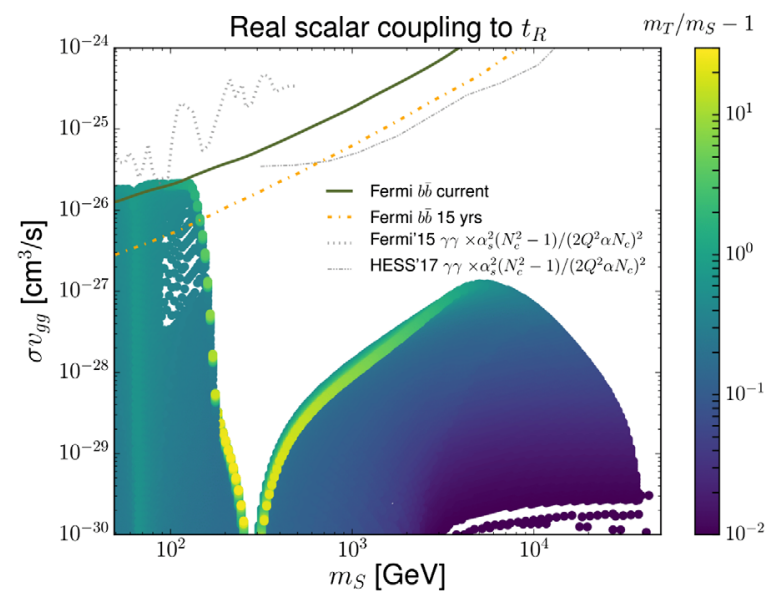

FIG. 6. Predictions for the $S S \rightarrow g g$ annihilation cross section, to which we superimpose the upper limits extracted from the cosmic-ray analysis of dwarf spheroidal galaxy data in the $b \bar{b}$ channel from Fermi-LAT [35] using current results (dark green continuous line) and projected results assuming 15 years of data acquisition (orange dot-dashed line) [33]. We also indicate the upper limits obtained from the gamma-ray line analysis of FermiLAT [36] and H.E.S.S. [34] by gray dotted and double-dotdashed lines, respectively (see the text for details). The color code represents the value of the $r-1$ parameter, and we have considered DM models satisfying the relic density constraints of Sec. III.

models with a DM abundance dominated by the resonant coannihilation of a TS system into a top quark.

We superimpose to our predictions limits extracted from varied observations. DM annihilations into top-antitop systems can be constrained with antiproton cosmic-ray data [32] (continuous green lines in Fig. 5). We also show Fermi-LAT gamma-ray constraints from dwarf spheroidal analysis for annihilations into a $b \bar{b}$ final state [35] (continuous dark green line in Fig. 6) and the corresponding prospects from 15 years of Fermi-LAT running [33] (dotdashed orange lines). While the Fermi-LAT Collaboration has not published any specific limits for what concerns the gamma-ray spectrum issued from DM annihilations into the $t \bar{t}$ and $g g$ final states, both spectra are expected to show a similar behavior as for annihilations into a $b \bar{b}$ system, as illustrated in Fig. 7 for $m_{S}$ below (upper panel) and above (lower panel) the top mass. An estimate of the limits for $t \bar{t}$ and $g g$ final states can be obtained following the methodology advocated in Ref. [37], using exclusion limits from DM annihilations into $b \bar{b}$ pairs that are rescaled using

$$
\sigma v_{g g, t \bar{t}}=\sigma v_{b \bar{b}} \frac{N_{\gamma}^{b \bar{b}}}{N_{\gamma}^{g g, t \bar{t}}},
$$

where $N_{\gamma}^{X}$ is the number of photon expected from an $X$ final state. We have nevertheless verified that $N_{\gamma}^{b \bar{b}}<N^{g g, t \bar{t}}$ by determining $N_{\gamma}^{X}$ using the hadronization model of PYTHIA8 [38], so the obtained bounds can be seen as conservative.
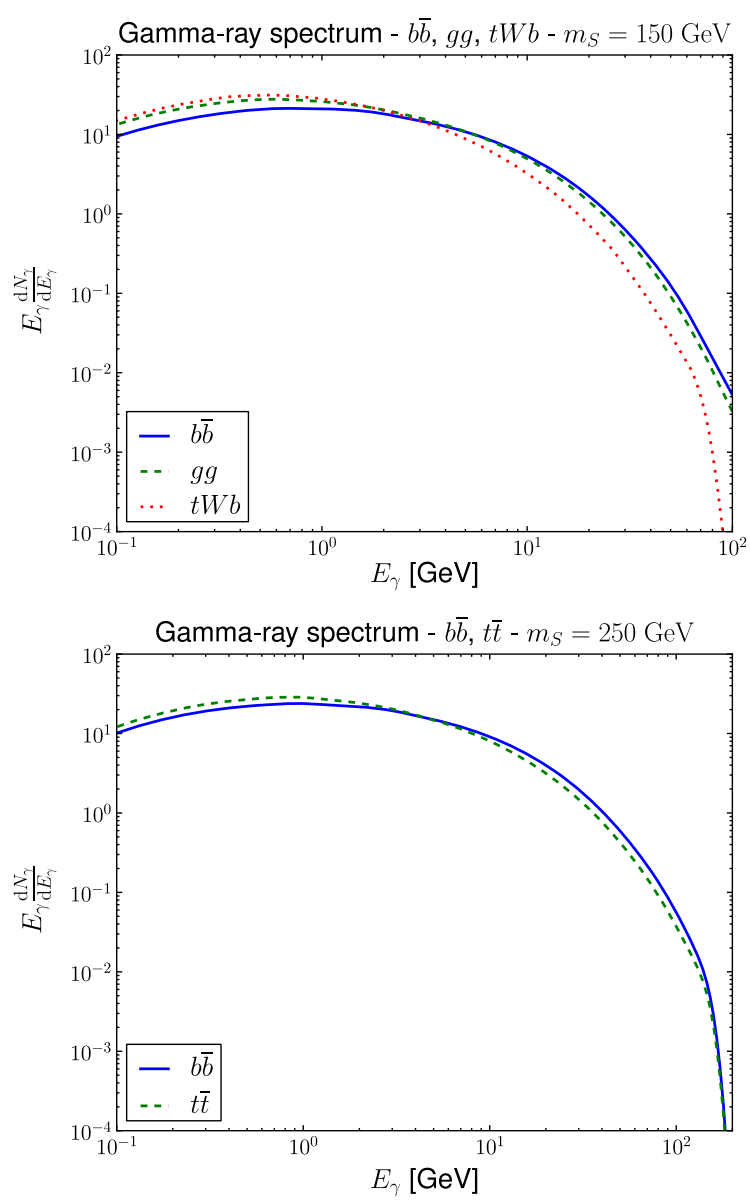

FIG. 7. Gamma-ray spectra as obtained with PYTHIA8 [38] for different DM annihilation mechanisms. We consider a DM mass of $m_{S}=150$ (upper panel) and $250 \mathrm{GeV}$ (lower panel).

The shape of the gamma-ray spectrum could also potentially be used to get hints on DM, as radiative corrections may give rise to a specific gamma-ray spectral imprint such as linelike features. However, these are most of the time overwhelmed by the continuum originating from the hadronization of the annihilation products. There are, however, two regimes in which they may be potentially important, namely, in the low mass range $\left(m_{S}<m_{t}\right)$ where annihilations into a photon pair could be relevant and in the multi$\mathrm{TeV}$ regime where radiative emission is crucial (as shown on the different panels of Fig. 5). The typical gamma-ray spectral signature of the annihilation of a pair of very heavy $S$ particles into $t \bar{t}, \gamma \gamma$, and $g g$ systems is presented in Fig. 8, our predictions being derived as sketched in Appendix C.

\section{1. $m_{S} \gtrsim 5 \mathrm{TeV}$}

The $m_{S} \gtrsim 5 \mathrm{TeV}$ regime is the one for which VIB emissions play a significant role and for which the approximation of Eq. (3.1) holds. DM annihilations into a top-antitop system produced in association with a photon can then be simply deduced, 

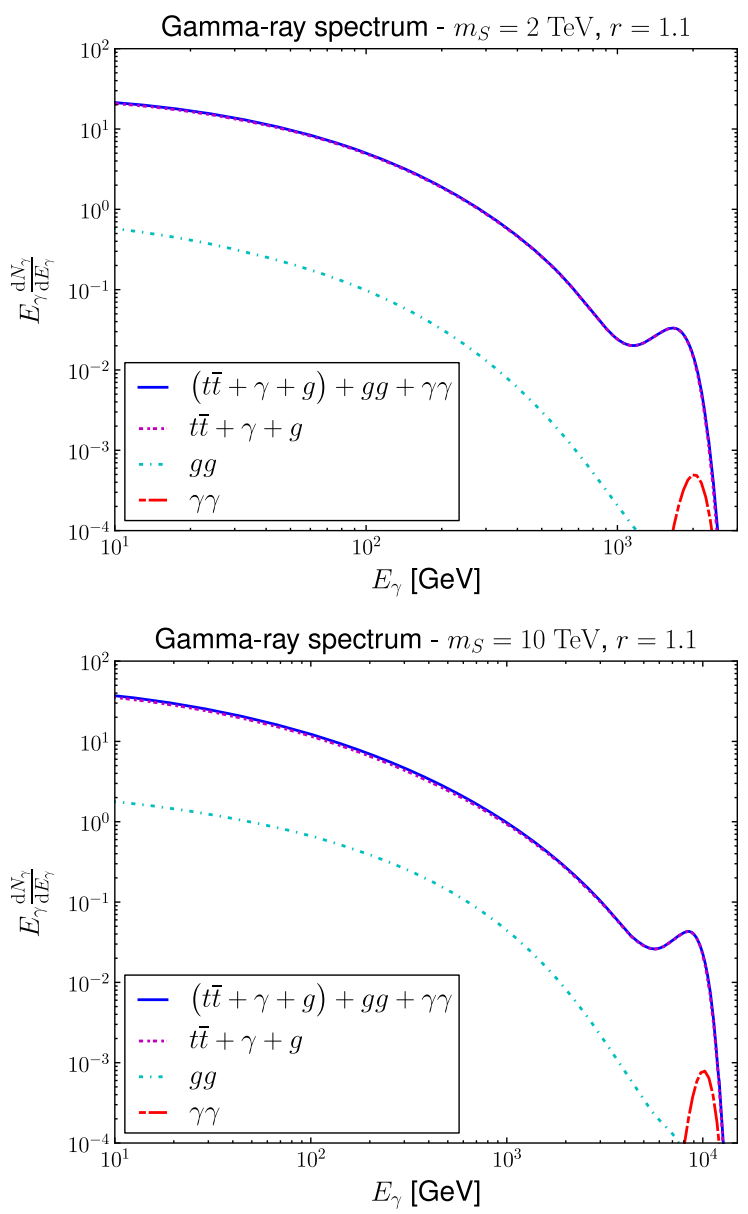

FIG. 8. Gamma-ray spectrum originating from the annihilation of a pair of $S$ particles of mass $m_{S}=2 \mathrm{TeV}$ (upper panel) and $10 \mathrm{TeV}$ (lower panel) and for a mediator mass fixed through the $r$ parameter that is set to $r=1.1$. Our predictions include (virtual and final-state) gluon and photon emissions from a $t \bar{t}$ final state as well as the direct one-loop contributions issued from annihilations into a pair of monochromatic photons and gluons.

$$
\frac{\sigma v_{t \bar{t} \gamma}}{\sigma v_{t \bar{t} g}}=\frac{2 N_{c} Q^{2} \alpha}{\left(N_{c}^{2}-1\right) \alpha_{s}} \approx 2.3 \times 10^{-2},
$$

where $N_{c}=3$ denotes the number of colors. Moreover, $\alpha$ and $\alpha_{s}$ stand for the electromagnetic and strong coupling constants, and we use $Z$-pole values as references, $\alpha=$ $1 / 128$ and $\alpha_{s}=0.112$. Although results from the H.E.S.S. Collaboration can potentially constrain the model, there is no official VIB dedicated analysis, and one must thus refer to the independent analysis of Ref. [39] and the recent constraints that can be extracted from the gamma-ray spectrum issued from the Galactic center [34]. This suggests that the annihilation cross section can be of at most $\sigma v_{i \overline{t y}} \sim 10^{-27} \mathrm{~cm}^{3} / \mathrm{s}$ for DM masses of about $10 \mathrm{TeV}$, which can be translated as $\sigma v_{t \bar{t} g} \sim 10^{-25} \mathrm{~cm}^{3} / \mathrm{s}$. This is illustrated in the lower panel of Fig. 5, in which we show the H.E.S.S. constraints derived in Ref. [34], after including both the rescaling factor of Eq. (4.5) and a factor of 2 accounting for the photon multiplicity.

$$
\text { 2. } m_{S}<m_{t}
$$

In the $m_{S}<m_{t}$ regime, $\sigma v_{g g}$ can be as large as about $2 \times 10^{-26} \mathrm{~cm}^{3} / s$ (see Fig. 6), and there is a well-defined prediction for annihilations into a pair of photons [40],

$$
\frac{\sigma v_{\gamma \gamma}}{\sigma v_{g g}}=\frac{4 Q^{4} \alpha^{2} N_{c}^{2}}{\alpha_{S}^{2}\left(N_{c}^{2}-1\right)} \approx 4.3 \times 10^{-3} .
$$

The strongest constraints on the production of gamma-ray lines at energies around and below $m_{t}$ originate from the Fermi-LAT Collaboration [36], and we indicate them in Fig. 6 after including the rescaling factor of Eq. (4.6) (gray dotted line). H.E.S.S. bounds at larger DM masses are also indicated, following Ref. [34] (double-dotted-dashed line). In both cases, we use the limits associated with an Einasto DM density profile.

To conclude this section, we project the DM indirect detection constraints from the cosmic-ray analysis (green region at large mass) and existing (dark green region at low mass) and future (orange region with a dotted-dashed contour) Fermi-LAT constraints from the gamma-ray continuum from dwarf spheroidal galaxies in the summary of Fig. 11. The color code is the same as in Figs. 5 and 6. A substantial part of the parameter space, for the $m_{S}<1 \mathrm{TeV}$ region, turns out to be constrained by probes of the gammaray continuum and antiproton cosmic rays. Moreover, for moderately heavy DM candidates, these constraints are complementary to those originating from direct DM searches studied in Sec. IVA. As for the relic density, annihilations into pairs of gluons are relevant for light DM $\left(m_{S}<m_{t}\right)$, while annihilations into top-antitop systems help us test heavier candidates with masses ranging up to $m_{S} \sim 400$ and $450 \mathrm{GeV}$ when observations based on gamma rays and antiprotons are, respectively, used. The major difference with the relic density considerations is that close to the top-antitop threshold the nonzero DM velocity at the freeze-out time allows for DM annihilations into a $t \bar{t}$ pair, which is kinematically forbidden today. A three-body $t W b$ final state, which does not yield further constraints, must therefore be considered instead. Finally, the predicted annihilation cross sections $\sigma v_{g g}$ and $\sigma v_{t \bar{t} g}$ appear to be too small to allow us to constrain the models using searches of specific features in the gamma-ray spectrum (considering an Einasto DM density profile).

\section{COLLIDER CONSTRAINTS}

Searches for new physics have played an important role in past, current, and future physics programs at colliders. In the context of the class of scenarios investigated in this work, in which the Standard Model is extended by a bosonic DM candidate and a fermionic vectorlike mediator, 
the results of many collider analyses can be reinterpreted to constrain the model.

In our model, the extra scalar particle is rendered stable (and thus a viable candidate for DM) by assuming a $\mathbb{Z}_{2}$ symmetry under which all new states are odd and all Standard Model states are even. As a consequence, the collider signatures of the model always involve final states containing an even number of odd particles that each decay into Standard Model particles and a DM state. This guarantees the presence of a large amount of missing transverse energy as a generic model signature.

For top-philic models, the relevant signatures can be classified into two classes, the model-independent mono-X searches that target the production of a pair of DM particles in association with a single energetic visible object $\mathrm{X}$, and the production of a pair of top-antitop quarks in association with missing energy.

Before going through the most recent constraints originating from LHC searches for DM, we will account for LEP results. In electron-positron collisions, top partners can be produced electroweakly,

$$
e^{+} e^{-} \rightarrow \gamma^{*}, \quad Z \rightarrow T \bar{T} \rightarrow t \bar{t}+E_{T},
$$

and yield a signature made of a pair of top-antitop quarks and missing transverse energy $\mathscr{E}_{T}$. Reinterpreting the results of the LEP searches for the supersymmetric partner of the top quark, vectorlike (top) partners are essentially excluded if their mass satisfies $m_{T} \lesssim 100 \mathrm{GeV}$ [41]. This excludes the lower left corner of the viable parameter space of the summary of Fig. 11 (magenta region) corresponding to DM masses of typically $m_{S}<78 \mathrm{GeV}$.

At the LHC, pairs of mediators can be copiously produced by virtue of the strong interaction. Top-antitop production in association with missing energy consists of the corresponding signature, as each mediator then decays, with a $100 \%$ branching fraction, into a system comprised of a top quark and a DM particle,

$$
p p \rightarrow T \bar{T} \rightarrow t S \bar{t} S
$$

Contributions to this process are illustrated by the first two Feynman diagrams of Fig. 9. Such a top-antitop plus
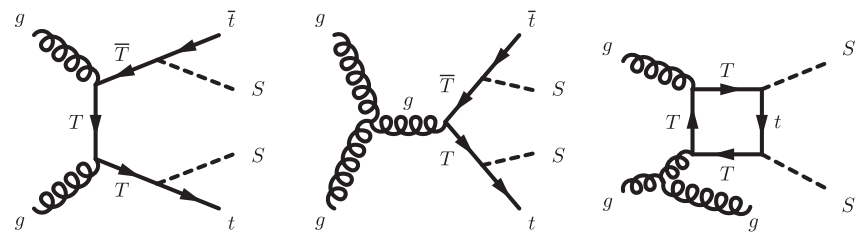

FIG. 9. Representative Feynman diagrams corresponding to the collider signatures of the model. We consider the pair production of vectorlike quarks that then decay each into a DM state and a top quark (leftmost and central diagrams) and loop-induced monojet production (rightmost diagram). missing energy signature has been widely studied by both the ATLAS and CMS collaborations, in particular in Run 2 searches for the superpartners of the top quark (assuming a decay into a top quarks and missing energy carried by a neutralino) [42-50] and in dedicated DM searches [51].

Additionally, the model can also be probed through classical DM searches using mono-X probes. Among all mono-X searches, we focus on the monojet one given the relative magnitude of the strong coupling with respect to the strength of the electroweak interactions. In this case, the considered signature exhibits the presence of a hard QCD jet recoiling against a large quantity of missing energy carried away by a pair of DM particles. Such a process,

$$
p p \rightarrow S S j,
$$

is loop induced in our model, as illustrated by the last Feynman diagram of Fig. 9. Although early monojet analyses were vetoing events featuring any extra hadronic activity through additional hard jets, it has been demonstrated that the latter could consist of useful handles to get a better sensitivity to the signal [52]. For this reason, recent ATLAS and CMS monojet analyses now include several signal regions in which more than one hard jet is allowed [53-56].

\section{A. Simulation details}

To reinterpret relevant results of the LHC in the context of the considered top-philic DM scenario and to determine their impact, we have implemented the Lagrangian of Eq. (2.1) into the FeYnRules program [57]. With the help of a joint usage of the NLOCT [58] and FeYnARTS [59] packages, we have analytically evaluated the ultraviolet and so-called $R_{2}$ counterterms required for numerical one-loop computations in four dimensions. The information has been exported under the form of a NLO Universal FeynRules Output model [60] containing, in addition to the tree-level model information, the $R_{2}$ and NLO counterterms.

We rely on the MADGRAPH 5_AMC@NLO [61] platform for the generation of hard-scattering events, at the NLO accuracy in QCD for the vectorlike quark pair production process of Eq. (5.2) and at the LO accuracy for the loopinduced monojet process of Eq. (5.3). In our simulation chain, we respectively convolute the LO and NLO matrix elements with the LO and NLO sets of NNPDF3.0 parton distribution functions [62], which we access through the LHAPDF6 library [63]. Moreover, the unphysical scales are always set to half the sum of the transverse mass of all finalstate particles.

The decay of the heavy $T$ quark into DM and a top quark,

$$
T \rightarrow t S
$$

is factorized from the production processes and is handled with the MADSPIN [64] and MADWIDTH [65] programs, 
together with those of all Standard Model heavy particles. For each considered new physics setup, we have consequently checked that the narrow-width approximation could be safely and consistently used, which is guaranteed by the fact that the mediator decay width satisfies $\Gamma_{T} / m_{T}<0.2$.

The resulting partonic events are matched with parton showers by relying on the PYTHIA8 code [38] and the MC@NLO prescription [66]. While hadronization is also taken care of by PYTHIA, we simulate the response of the ATLAS and CMS detectors by means of the DELPHES3 program [67] that internally relies on the anti- $k_{T}$ jet algorithm [68] as implemented in the FASTJET software [69] for object reconstruction. For each of the analyses that we have recast, the DELPHES configuration has been tuned to match the detector setup described in the experimental documentation. We have used the MADANALYsis5 framework [70-72] to calculate the signal efficiencies for the different considered search strategies and to derive 95\% C.L. exclusions with the $\mathrm{CL}_{s}$ method [73].

\section{B. Reinterpreted LHC analyses}

To assess the reach of LHC searches for DM in topantitop quark production in association with missing energy $\left(p p \rightarrow t \bar{t}+E_{T}\right)$, we reinterpret a CMS analysis of collision events featuring a pair of leptons of opposite electric charge [50]. While other final states in the single lepton and fully hadronic decay mode of the top-antitop pair are relevant as well, all these LHC searches are so far found to yield similar bounds. For this reason, we have chosen to focus on a single of those channels, namely, the cleaner dileptonic decay mode of the topantitop pair.

The CMS-SUS-17-001 analysis of Ref. [50] focuses on the analysis of $35.9 \mathrm{fb}^{-1}$ of LHC collisions featuring the presence of a system of two isolated leptons of opposite electric charges, which is compatible neither with a lowmass hadronic resonance nor with a $Z$ boson. One requires the presence of a large amount of missing transverse energy, as well as of at least two hard jets with at least one of them being $b$ tagged. The latter is required to possess a large significance and to be well separated from the two leading jets. After this preselection, the analysis defines three aggregated signal regions depending on the value of the missing energy and of the transverse mass $m_{T 2}[74,75]$ reconstructed from the two leptons and the missing momentum.

In addition, we include in our investigations the CMSSUS-16-052 analysis, which is dedicated to probing the more compressed regions of the parameter space with $35.9 \mathrm{fb}^{-1}$ of LHC collisions [76]. In this analysis, it is assumed that the top partner cannot decay on shell into a top quark plus missing energy system, so the search strategy is optimized for top partners decaying into systems made of three mostly soft fermions (including $b$ quarks) and missing energy via an off-shell top quark. Event selection requires the presence of one hard initial-state-radiation jet and of at most a second jet well separated from the first one. Moreover, one asks for a single identified lepton, a large amount of missing energy, and an important hadronic activity. The threshold values that are imposed and the detailed properties of the lepton, the missing energy, and the hadronic activity allow us to define two classes of three signal regions targeting varied new physics configurations.

We have also compared predictions originating from the simulation of the process of Eq. (5.2) with the 8 LHC Run 1 results, and we have in particular assessed the compatibility with the null results of the $8 \mathrm{TeV}$ search labeled CMS-B2G14-004 [77-79]. This search focuses on singly leptonic final states containing at least three jets (including at least one $b$-tagged jet) and a large amount of missing energy well separated from the jets. The event selection moreover constrains the transverse mass of the system comprising the lepton and of the missing transverse momentum, as well as the $m_{T 2}^{W}$ transverse variable [80].

For the reinterpretation of the LHC search results for mono-X DM signals, we have considered two ATLAS analyses targeting a monojetlike topology, i.e., at least one very hard jet recoiling against some missing momentum and a subleading jet activity. Although those analyses $[53,81]$ focus on a small integrated luminosity of LHC collisions $\left(3.2 \mathrm{fb}^{-1}\right)$, they are already limited by the systematics so that the constraints derived from early Run 2 data are not expected to get more severe in the future [82]. In the ATLAS-EXOT-2015-03 analysis $[53,83]$, the target consists in a monojetlike topology in which the subleading jet activity is rather limited, the event selection being allowed to contain only up to three additional jets. Seven inclusive and seven exclusive signal regions are defined, the differences between them being related to various requirements on the missing energy. In contrast, the ATLAS-SUSY-2015-06 analysis [81,84] allows for both a small and larger subleading jet activity, the event selection being dedicated to final states containing two to six jets. Seven signal regions are defined, depending on the number and on the kinematic properties of the jets and on the missing momentum.

All the above analyses are implemented and validated in the MADANALYSIS5 framework and have thus been straightforwardly and automatically used within the simulation chain depicted in Sec. VA. We consider a new physics signal including contributions from both processes of Eqs. (5.2) and (5.3), although vectorlike quark pair production largely dominates for perturbative $\tilde{y}_{t}$ values.

\section{Collider constraints}

In Fig. 10, we report our findings in the $\left(m_{T}, m_{S}\right)$ mass plane. As the vectorlike-quark production process of Eq. (5.2) dominates regardless of the actual value of the $\tilde{y}_{t}$ coupling, the latter is irrelevant for what concerns constraints stemming from the LHC. This is induced by 


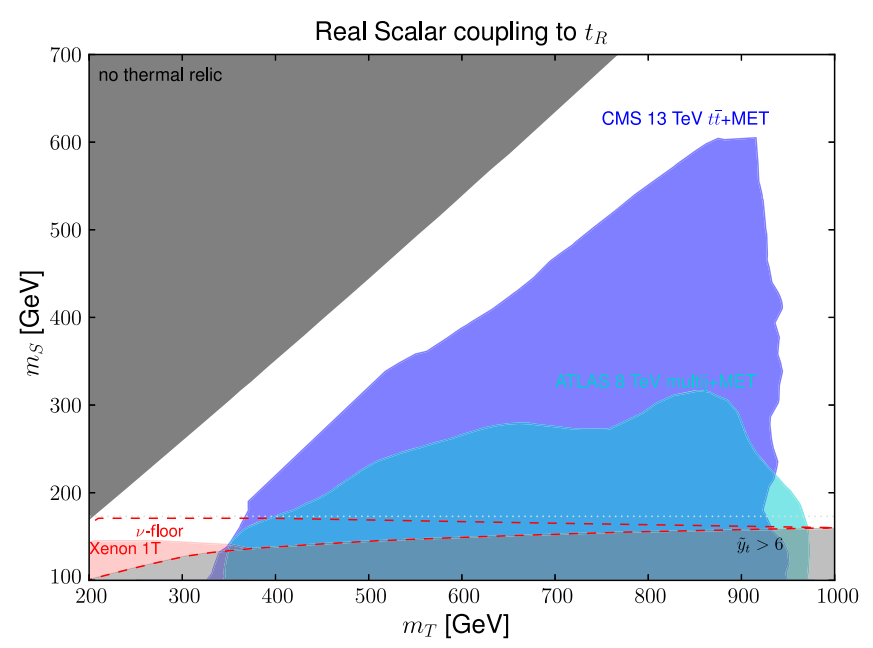

FIG. 10. Collider constraints on our top-philic DM model expressed, together with the relic density and DM direct detection bounds, in the $m_{S}, m_{T}$ mass plane.

the fact that the vectorlike mediator only couples to the top quark, which contrasts with scenarios in which couplings to lighter quarks exist. Those interactions with the first- and second-generation SM quarks indeed yield extra contributions featuring a direct dependence on the Yukawa couplings [5]. Coming back to the considered top-philic scenario, all results can thus be represented in the $\left(m_{S}\right.$, $\left.m_{S} / m_{T}-1\right)$ two-dimensional plane. In the figure, we superimpose to the cosmology considerations discussed in the previous sections (namely, the relic density and direct detection bounds, the indirect bounds not being reproduced, so as to avoid cluttering of the figure) the constraints that can be obtained by reinterpreting the results of the LHC searches for new physics discussed in Sec. V B. The white region corresponds to configurations for which the experimentally observed relic abundance is reproduced and that are not excluded by current cosmological data. In the (excluded) light gray region, a correct abundance would imply going beyond the perturbative regime, while in the dark gray region, the dark matter particle $S$ is unstable as not the lightest $\mathbb{Z}_{2}$-odd particle.

For each new physics configuration and each signal region of each considered analysis, we evaluate the number of signal events $s$ surviving the selection with MADANALYSIS5 and extract a $\mathrm{CL}_{s}$ exclusion from the observed number of events $n_{\text {data }}$ populating the region and the expected number of background events $\hat{b} \pm \Delta b$. To this aim, we undertake 100.000 toy experiments in which we generate the actual number of background events $b$ by assuming that the corresponding distribution is a Gaussian of mean $\hat{b}$ and width $\Delta b$. We then consider two Poisson distributions of parameters $b$ and $b+s$ to evaluate the $p$ values of the signal-plus-background and background-only hypotheses, knowing that $n_{\text {data }}$ events have been observed. From these $p$ values, we derive the associated $\mathrm{CL}_{s}$ value.
The colored regions shown in Fig. 10 are excluded at the 95\% C.L. by at least one signal region of the considered analyses. The dark blue region corresponds to what we obtain with the reinterpretation of the results of the two CMS searches for DM in the top-antitop plus missing energy channel, namely, CMS-SUS-17-001 and CMSSUS-16-052. While our results only focus on Run 2 data, we have verified that the obtained limits are compatible with the less stringent Run 1 constraints derived from the results of the CMS-B2G-14-004 analysis. The light blue area depicted in the figure corresponds to bounds that can be extracted from the reinterpretation of the results of the ATLAS-EXOT-2015-03 and ATLAS-SUSY-2015-06 searches for new physics in the multijet plus missing energy channel.

We have found that mediator masses ranging up to $1 \mathrm{TeV}$ are excluded, provided that the DM mass is light enough for having enough phase space to guarantee the decay of the mediator into a DM particle and a top quark in a far-from-threshold regime. While generic multijet plus missing energy searches are quite sensitive when the DM mass is small, they quickly lose any sensitivity for larger $m_{S}$ values. This stems from the monojetlike selection of the considered analyses, which can only be satisfied if enough phase space is available for the $T$ decay process.

As soon as the $T \rightarrow t S$ decay channel is closed, the $T$ quark becomes long lived enough to hadronize before decaying, and it could potentially travel on macroscopic distances in the detector. While the unknown modeling of vectorlike quark hadronization would introduce uncontrolled uncertainties on the predictions, none of the currently available computer tools allows for a proper handling of long-lived colored particles. Moreover, all considered LHC analyses have been designed for being sensitive to promptly decaying new-physics states and are thus expected to lose sensitivity when new physics particles are long lived. For this reason, we restrict ourselves to providing LHC constraints in the region of the parameter space where the $T$ quark can promptly decay into a top quark and a DM particle.

\section{SUMMARY}

The WIMP paradigm is being tested by various experiments, in astrophysics and cosmology and at colliders. At the same time, there is a significant interest on top-philic new physics, as the top quark is widely considered, due to its large mass, as a perfect laboratory for the study of the electroweak symmetry breaking mechanism. In this work, we have extensively investigated a simple DM scenario that naturally brings these topics together. It is based on a real scalar particle coupled to the top quark through a Yukawa coupling with a heavy vectorlike quark. As in the SM sector the top quark has the largest coupling to the Higgs boson, it is at least conceivable that it also features the largest coupling to a new dark sector. The model rests only on 


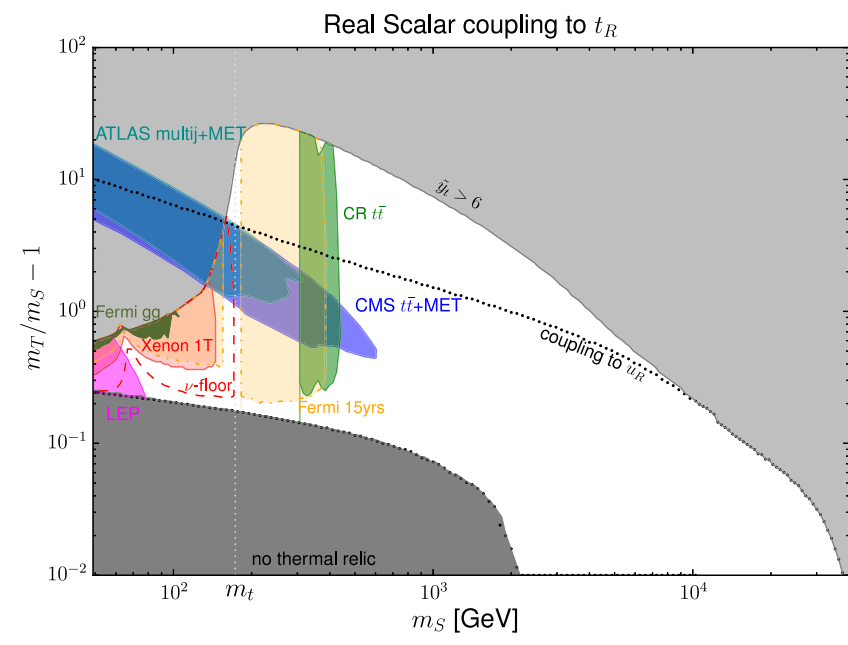

FIG. 11. Phenomenologically viable region of our model parameter space, presented in the $\left(m_{S}, r-1\right)$ plane, on which we project constraints from DM direct and indirect detection and collider experiments. The grey regions correspond to regions for which the relic density cannot be accommodated. Direct DM searches: The red region is excluded by the Xenon 1T [29] experiment at the $90 \%$ confidence level, while the region delimited by the red dashed line is in principle testable by DM direct detection searches as lying above the neutrino floor [31]. Indirect DM searches: the dark green (at low mass) and light green (at large mass) regions are excluded by Fermi-LAT gamma-ray constraints [35] and by the cosmic-ray (CR) analysis of Ref. [32]. The orange region delimited by a dotted-dashed line is the projected sensitivity of Fermi-LAT after 15 years of exposure [33]. Collider searches: constraints on top partner production at LEP [41] and the LHC [50,76] are, respectively, shown by the magenta and light blue regions, while mono-X bounds $[53,81]$ are indicated by the dark blue region.

very few parameters (one coupling strength and two masses), so it provides a good starting point to compare the impact of different experimental results from varied origins. In the present case, we focus on DM direct and indirect detection searches, as well as on collider probes. We have studied the constraints on the DM model, paying special attention to the potential impact of the QCD radiative corrections on all the considered bounds (i.e., the DM relic abundance, the DM direct and indirect searches, and the collider searches). In this way, our study complements and extends similar earlier works based on Majorana DM candidates [14,15,85].

Our analysis reveals that, although there is a complementarity between the different searches, only a small fraction of the viable parameter space of this very simple DM scenario is tested by the current experiments. This is illustrated in Fig. 11, which summarizes our results and complements the information provided in Fig. 10. In the long term, the most fruitful strategy to further test such a DM scenario would be to increase the energy reach at colliders.

\section{ACKNOWLEDGMENTS}

We thank J. Heisig and M. Korsmeier for enlightening discussions and P. Wu for useful comments on our work. This study has been partly supported by French state funds managed by the Agence Nationale de la Recherche (ANR) in the context of the LABEX ILP (Grants No. ANR-11IDEX-0004-02 and No. ANR-10-LABX-63), the Fonds pour la formation à la Recherche dans l'Industrie et dans l'Agriculture (FRIA), the Fonds de la Recherche Scientifique-FNRS, the Strategic Research Program High Energy Physics and the Research Council of the Vrije Universiteit Brussel, the IISN convention 4.4503.15, and the Excellence of Science convention 30820817.

\section{APPENDIX A: SOMMERFELD CORRECTIONS TO THE RELIC ABUNDANCE}

DM annihilation can proceed directly through the

$$
S S \rightarrow t \bar{t}^{(*)}, \quad g g \quad \text { and } \quad t \bar{t} g
$$

channels. If the vectorlike fermionic mediator is not too heavy relative to the DM particle, it may still be abundant at the time of freeze-out and hence either annihilates or coannihilates [86],

$$
\begin{aligned}
& S T \rightarrow g t, \quad S \bar{T} \rightarrow g \bar{t}, \\
& T \bar{T} \rightarrow g g \quad \text { or } \quad q \bar{q}, \\
& T T \rightarrow t t, \quad \bar{T} \bar{T} \rightarrow \bar{t} \bar{t},
\end{aligned}
$$

with the latter processes involving $t$-channel DM exchanges. The $T \bar{T}, T T$, and $\bar{T} \bar{T}$ channels are impacted by either attractive or repulsive Sommerfeld effects through gluonic exchanges [87]. To account for these effects, we have followed the procedure depicted in Ref. [5] with the only difference being that we have taken explicitly into account the top-quark mass effects on the annihilation cross sections. In addition, we have verified, through various approaches, that our treatment is correct in the $s$-wave approximation. Our treatment agrees with the results of Ref. [88] in the $s$-wave approximation. Going beyond this approximation is, however, known to lead to corrections to the relic density of less than $1 \%$, as shown, e.g., in supersymmetry [89].

On general grounds, we should also take into account the possible formation of $T \bar{T}, T T$, and $\bar{T} \bar{T}$ bound states, which would imply a modification of the Sommerfeld corrections. It has, however, been concluded, using a setup similar to ours, that bound states have only a moderate impact on the DM relic density $[90,91]$. As the Sommerfeld corrections affect the relic abundance by less than $15 \%$, we ignore bound-state formation from the present calculations. 


\section{APPENDIX B: $S S \rightarrow g g$ CROSS SECTION}

Analytical expressions for the $S S \rightarrow g g(\gamma \gamma)$ annihilation cross section have been given in Refs. [3,4] in terms of one-loop three-points functions. Correcting a typo in Ref. [3], the cross section $\sigma v$ for DM annihilation into a gamma or gluon pair is given by

$$
\sigma v=\frac{2 \tilde{y}_{t}^{4}}{64 \pi^{3} m_{S}^{2}} \begin{cases}Q^{4} \alpha^{2} N_{c}^{2}|\mathcal{M}|^{2} & \text { for photons } \\ \frac{\alpha_{S}^{2}\left(N_{c}^{2}-1\right)}{4}|\mathcal{M}|^{2} & \text { for gluons. }\end{cases}
$$

In this expression, the one-loop amplitude reads

$$
\begin{aligned}
\mathcal{M}= & 2+\left\{\frac{1-r^{2}-z^{2}}{1+r^{2}-z^{2}} \frac{2 z^{2}}{r^{2}-z^{2}}\right. \\
& \times C_{0}\left(-m_{S}^{2}, m_{S}^{2}, 0 ; z^{2} m_{S}^{2}, r^{2} m_{S}^{2}, z^{2} m_{S}^{2}\right)+\frac{4 z^{2}\left(1-z^{2}\right)}{1+r^{2}-z^{2}} \\
& \left.\times C_{0}\left(4 m_{S}^{2}, 0,0 ; z^{2} m_{S}^{2}, z^{2} m_{S}^{2},{ }^{2} z m_{S}^{2}\right)+z \leftrightarrow r\right\}, \quad
\end{aligned}
$$

where $z=\frac{m_{t}}{m_{S}}$ and

$$
\begin{aligned}
& C_{0}\left(p_{1}^{2},\left(p_{1}-p_{2}\right)^{2}, p_{2}^{2} ; m_{1}^{2}, m_{2}^{2}, m_{3}^{2}\right) \\
& =\int \frac{\mathrm{d}^{4} l}{i \pi^{2}} \frac{1}{l^{2}-m_{1}^{2}} \frac{1}{\left(l+p_{1}\right)^{2}-m_{2}^{2}} \frac{1}{\left(l+p_{2}\right)^{2}-m_{3}^{2}} .
\end{aligned}
$$

The scalar three-point functions are written as [92]

$$
\begin{aligned}
C_{0}\left(4 m_{S}^{2}, 0,0 ; z^{2} m_{S}^{2}, z^{2} m_{S}^{2}, z^{2} m_{S}^{2}\right) & =\frac{1}{4} I_{1}\left(1, z^{2}\right), \\
C_{0}\left(-m_{S}^{2}, m_{S}^{2}, 0 ; z^{2} m_{S}^{2}, r^{2} m_{S}^{2}, z^{2} m_{S}^{2}\right) & =-\frac{1}{2} I_{2}\left(\frac{1}{z^{2}}, \frac{r^{2}}{z^{2}}\right),
\end{aligned}
$$

with

$$
I_{1}\left(1, z^{2}\right)=\left\{\begin{array}{l}
\frac{1}{2}\left[\left(\log \frac{1+\sqrt{1-z^{2}}}{1-\sqrt{1-z^{2}}}\right)^{2}-\pi^{2}\right] \quad \text { if } \mathrm{z}<1 \\
-2\left(\arctan \frac{1}{\sqrt{z^{2}-1}}\right)^{2} \text { if } \mathrm{z}>1
\end{array}\right.
$$

and

$$
\begin{aligned}
& I_{2}\left(\frac{1}{z^{2}}, \frac{r^{2}}{z^{2}}\right) \\
& =\mathrm{Li}_{2}\left(\frac{1-r^{2}+z^{2}-\sqrt{s_{1}}}{2 z^{2}}\right)+\mathrm{Li}_{2}\left(\frac{1-r^{2}+z^{2}+\sqrt{s_{2}}}{2 z^{2}}\right) \\
& \quad-\mathrm{Li}_{2}\left(\frac{1-r^{2}+z^{2}-\sqrt{s_{3}}}{2 z^{2}}\right)-\mathrm{Li}_{2}\left(\frac{-1-r^{2}+z^{2} \sqrt{s_{4}}}{2 z^{2}}\right) .
\end{aligned}
$$

In those expressions, we have introduced the variables

$$
\begin{aligned}
& s_{1}=\left(1+r^{2}-z^{2}\right)^{2}-4 r^{2}, \\
& s_{2}=\left(1-r^{2}-z^{2}\right)^{2}-4 r^{2} z^{2}, \\
& s_{3}=s_{4}=\left(1+r^{2}+z^{2}\right)^{2}-4 r^{2} z^{2} .
\end{aligned}
$$

\section{APPENDIX C: ON QCD CORRECTIONS TO THE $S S \rightarrow \bar{t} g(\gamma)$}

In this section, we comment on our derivation of the differential cross section for the $S S \rightarrow t \bar{t} g(\gamma)$ process at the partonic level and on the methods that have been used to cope with the hadronization of the colored final-state particles.

At $\mathcal{O}\left(\alpha_{s}\right)$, the analytical expression for the $S S \rightarrow t \bar{t} g$ amplitude includes contributions of gluon emission by the final-state quarks and the intermediate particle $T$. When relevant, final-state radiation typically gives rise to double Sudakov logarithms associated with soft and collinear divergences, which must be consistently taken into account, in particular in regimes where they are large.

In our model, VIB emission is finite and moreover only relevant for the radiation of highly energetic gluons. When VIB contributions are negligible (i.e., for sufficiently large $m_{T} / m_{S}$ ratios), we have simply discarded them and used PYTHIA8 [38] to handle both final-state radiation and hadronization. This effectively resums the large logarithms via the use of an appropriate Sukadov form factor. When VIB contributions are relevant, the corresponding threebody hard-scattering process has been explicitly evaluated with CALCHEP [20], and we have made use of PytHiA8 to simulate the subsequent hadronization. Finally, for low energies, we have restricted our computation to the twobody $S S \rightarrow t \bar{t}$ process and relied on PYTHIA8 for the simulation of final-state radiation and hadronization. The matching of the separate contributions to the gamma-ray spectrum has been achieved by implementing an explicit cutoff on the gluon energy at the partonic level. More details can be found in Ref. [6], which is similar in spirit but differs in details from the prescription proposed in Ref. [15]. 
[1] T. Toma, Internal Bremsstrahlung Signature of Real Scalar Dark Matter and Consistency with Thermal Relic Density, Phys. Rev. Lett. 111, 091301 (2013).

[2] F. Giacchino, L. Lopez-Honorez, and M. H. G. Tytgat, Scalar dark matter models with significant internal bremsstrahlung, J. Cosmol. Astropart. Phys. 10 (2013) 025.

[3] F. Giacchino, L. Lopez-Honorez, and M. H. G. Tytgat, Bremsstrahlung and gamma ray lines in 3 scenarios of dark matter annihilation, J. Cosmol. Astropart. Phys. 08 (2014) 046.

[4] A. Ibarra, T. Toma, M. Totzauer, and S. Wild, Sharp gammaray spectral features from scalar dark matter annihilations, Phys. Rev. D 90, 043526 (2014).

[5] F. Giacchino, A. Ibarra, L. Lopez Honorez, M. H. G. Tytgat, and S. Wild, Signatures from scalar dark matter with a vector-like quark mediator, J. Cosmol. Astropart. Phys. 02 (2016) 002.

[6] S. Colucci, F. Giacchino, M. H. G. Tytgat, and J. Vandecasteele, On radiative corrections to vector-like portal dark matter, arXiv:1805.10173.

[7] T. Bringmann, F. Calore, A. Galea, and M. Garny, Electroweak and Higgs boson internal bremsstrahlung: General considerations for Majorana dark matter annihilation and application to MSSM neutralinos, J. High Energy Phys. 09 (2017) 041.

[8] J. Hisano, K. Ishiwata, and N. Nagata, Gluon contribution to the dark matter direct detection, Phys. Rev. D 82, 115007 (2010).

[9] S. Kraml, U. Laa, L. Panizzi, and H. Prager, Scalar versus fermionic top partner interpretations of $t \bar{t}+E_{T}^{\text {miss }}$ searches at the LHC, J. High Energy Phys. 11 (2016) 107.

[10] M. Chala, Direct bounds on heavy toplike quarks with standard and exotic decays, Phys. Rev. D 96, 015028 (2017).

[11] B. Fuks and H.-S. Shao, QCD next-to-leading-order predictions matched to parton showers for vector-like quark models, Eur. Phys. J. C 77, 135 (2017).

[12] S. Baek, P. Ko, and P. Wu, Top-philic scalar dark matter with a vector-like fermionic top partner, J. High Energy Phys. 10 (2016) 117.

[13] S. Baek, P. Ko, and P. Wu, Heavy quarks flavored real scalar dark matter, J. Cosmol. Astropart. Phys. 07 (2018) 008.

[14] M. Garny, J. Heisig, M. Hufnagel, and B. Lulf, Top-philic dark matter within and beyond the WIMP paradigm, Phys. Rev. D 97, 075002 (2018).

[15] T. Bringmann, A. J. Galea, and P. Walia, Leading QCD corrections for indirect dark matter searches: A fresh look, Phys. Rev. D 93, 043529 (2016).

[16] A. Birkedal, K. T. Matchev, M. Perelstein, and A. Spray, Robust gamma ray signature of WIMP dark matter, arXiv: hep-ph/0507194.

[17] M. Drees and K.-i. Hikasa, Note on QCD corrections to hadronic Higgs decay, Phys. Lett. B 240, 455 (1990).

[18] M. Drees and K.-i. Hikasa, Heavy quark thresholds in Higgs physics, Phys. Rev. D 41, 1547 (1990).

[19] G. Bélanger, F. Boudjema, A. Pukhov, and A. Semenov, micrOMEGAs4.1: Two dark matter candidates, Comput. Phys. Commun. 192, 322 (2015).
[20] A. Belyaev, N. D. Christensen, and A. Pukhov, CalcHEP 3.4 for collider physics within and beyond the Standard Model, Comput. Phys. Commun. 184, 1729 (2013).

[21] P. A. R. Ade et al. (Planck Collaboration), Planck 2013 results. XVI. Cosmological parameters, Astron. Astrophys. 571, A16 (2014).

[22] J. Edsjo and P. Gondolo, Neutralino relic density including coannihilations, Phys. Rev. D 56, 1879 (1997).

[23] M. Garny, J. Heisig, B. Lülf, and S. Vogl, Coannihilation without chemical equilibrium, Phys. Rev. D 96, 103521 (2017).

[24] J. M. Cline, K. Kainulainen, P. Scott, and C. Weniger, Update on scalar singlet dark matter, Phys. Rev. D 88, 055025 (2013).

[25] P. Athron et al. (GAMBIT Collaboration), Status of the scalar singlet dark matter model, Eur. Phys. J. C 77, 568 (2017).

[26] P. Gondolo and S. Scopel, On the sbottom resonance in dark matter scattering, J. Cosmol. Astropart. Phys. 10 (2013) 032.

[27] M. Drees and M. Nojiri, Neutralino-nucleon scattering revisited, Phys. Rev. D 48, 3483 (1993).

[28] J. Hisano, R. Nagai, and N. Nagata, Effective theories for dark matter nucleon scattering, J. High Energy Phys. 05 (2015) 037.

[29] E. Aprile et al. (XENON Collaboration), First Dark Matter Search Results from the XENON1T Experiment, Phys. Rev. Lett. 119, 181301 (2017).

[30] E. Aprile et al. (XENON Collaboration), Physics reach of the XENON1T dark matter experiment, J. Cosmol. Astropart. Phys. 04 (2016) 027.

[31] J. Billard, L. Strigari, and E. Figueroa-Feliciano, Implication of neutrino backgrounds on the reach of next generation dark matter direct detection experiments, Phys. Rev. D 89, 023524 (2014).

[32] A. Cuoco, J. Heisig, M. Korsmeier, and M. Krämer, Constraining heavy dark matter with cosmic-ray antiprotons, J. Cosmol. Astropart. Phys. 04 (2018) 004.

[33] E. Charles et al. (FERMI-LAT Collaboration), Sensitivity projections for dark matter searches with the Fermi Large Area Telescope, Phys. Rep. 636, 1 (2016).

[34] L. Rinchiuso, E. Moulin, A. Viana, C. Van Eldik, and J. Veh (H.E.S.S. Collaboration), Dark matter gamma-ray line searches toward the Galactic Center halo with H.E.S.S. I, Proc. Sci., ICRC2017 (2017) 893.

[35] M. Ackermann et al. (FERMI-LAT Collaboration), Searching for Dark Matter Annihilation from Milky Way Dwarf Spheroidal Galaxies with Six Years of Fermi Large Area Telescope Data, Phys. Rev. Lett. 115, 231301 (2015).

[36] M. Ackermann et al. (FERMI-LAT Collaboration), Updated search for spectral lines from Galactic dark matter interactions with pass 8 data from the Fermi Large Area Telescope, Phys. Rev. D 91, 122002 (2015).

[37] T. Bringmann, X. Huang, A. Ibarra, S. Vogl, and C. Weniger, Fermi LAT search for internal bremsstrahlung signatures from dark matter annihilation, J. Cosmol. Astropart. Phys. 07 (2012) 054.

[38] T. Sjöstrand, S. Ask, J. R. Christiansen, R. Corke, N. Desai, P. Ilten, S. Mrenna, S. Prestel, C. O. Rasmussen, and 
P.Z. Skands, An introduction to PYTHIA 8.2, Comput. Phys. Commun. 191, 159 (2015).

[39] A. Ibarra, H. M. Lee, S. Lopez Gehler, W.-I. Park, and M. Pato, Gamma-ray boxes from axion-mediated dark matter, J. Cosmol. Astropart. Phys. 05 (2013) 016.

[40] X. Chu, T. Hambye, T. Scarna, and M. H. G. Tytgat, What if dark matter gamma-ray lines come with gluon lines?, Phys. Rev. D 86, 083521 (2012).

[41] G. Abbiendi et al. (OPAL Collaboration), Search for scalar top and scalar bottom quarks at LEP, Phys. Lett. B 545, 272 (2002).

[42] M. Aaboud et al. (ATLAS Collaboration), Search for topsquark pair production in final states with one lepton, jets, and missing transverse momentum using $36 \mathrm{fb}^{-1}$ of $\sqrt{s}=$ $13 \mathrm{TeV}$ pp collision data with the ATLAS detector, J. High Energy Phys. 06 (2018) 108.

[43] M. Aaboud et al. (ATLAS Collaboration), Search for a scalar partner of the top quark in the jets plus missing transverse momentum final state at $\sqrt{s}=13 \mathrm{TeV}$ with the ATLAS detector, J. High Energy Phys. 12 (2017) 085.

[44] M. Aaboud et al. (ATLAS Collaboration), Search for supersymmetry in events with $b$-tagged jets and missing transverse momentum in $p p$ collisions at $\sqrt{s}=13 \mathrm{TeV}$ with the ATLAS detector, J. High Energy Phys. 11 (2017) 195.

[45] M. Aaboud et al. (ATLAS Collaboration), Search for direct top squark pair production in final states with two leptons in $\sqrt{s}=13 \mathrm{TeV} p p$ collisions with the ATLAS detector, Eur. Phys. J. C 77, 898 (2017).

[46] M. Aaboud et al. (ATLAS Collaboration), Search for supersymmetry in final states with two same-sign or three leptons and jets using $36 \mathrm{fb}^{-1}$ of $\sqrt{s}=13 \mathrm{TeV} p p$ collision data with the ATLAS detector, J. High Energy Phys. 09 (2017) 084.

[47] A. M. Sirunyan et al. (CMS Collaboration), Search for new phenomena with the $M_{\mathrm{T} 2}$ variable in the all-hadronic final state produced in proton-proton collisions at $\sqrt{s}=13 \mathrm{TeV}$, Eur. Phys. J. C 77, 710 (2017).

[48] A. M. Sirunyan et al. (CMS Collaboration), Search for direct production of supersymmetric partners of the top quark in the all-jets final state in proton-proton collisions at $\sqrt{s}=13$ TeV, J. High Energy Phys. 10 (2017) 005.

[49] A. M. Sirunyan et al. (CMS Collaboration), Search for top squark pair production in pp collisions at $\sqrt{s}=13 \mathrm{TeV}$ using single lepton events, J. High Energy Phys. 10 (2017) 019.

[50] A. M. Sirunyan et al. (CMS Collaboration), Search for top squarks and dark matter particles in opposite-charge dilepton final states at $\sqrt{s}=13 \mathrm{TeV}$, Phys. Rev. D 97, 032009 (2018).

[51] A. M. Sirunyan et al. (CMS Collaboration), Search for dark matter produced in association with heavy-flavor quark pairs in proton-proton collisions at $\sqrt{s}=13 \mathrm{TeV}$, Eur. Phys. J. C 77, 845 (2017).

[52] O. Buchmueller, S. A. Malik, C. McCabe, and B. Penning, Constraining Dark Matter Interactions with Pseudoscalar and Scalar Mediators Using Collider Searches for Multijets plus Missing Transverse Energy, Phys. Rev. Lett. 115, 181802 (2015).
[53] M. Aaboud et al. (ATLAS Collaboration), Search for new phenomena in final states with an energetic jet and large missing transverse momentum in $p p$ collisions at $\sqrt{s}=$ $13 \mathrm{TeV}$ using the ATLAS detector, arXiv:1604.07773.

[54] M. Aaboud et al. (ATLAS Collaboration), Search for dark matter and other new phenomena in events with an energetic jet and large missing transverse momentum using the ATLAS detector, J. High Energy Phys. 01 (2018) 126.

[55] A. M. Sirunyan et al. (CMS Collaboration), Search for dark matter produced with an energetic jet or a hadronically decaying $\mathrm{W}$ or $\mathrm{Z}$ boson at $\sqrt{s}=13 \mathrm{TeV}$, J. High Energy Phys. 07 (2017) 014.

[56] A. M. Sirunyan et al. (CMS Collaboration), Search for new physics in final states with an energetic jet or a hadronically decaying $\mathrm{W}$ or $\mathrm{Z}$ boson and transverse momentum imbalance at $\sqrt{s}=13 \mathrm{TeV}$, Phys. Rev. D 97, 092005 (2016).

[57] A. Alloul, N. D. Christensen, C. Degrande, C. Duhr, and B. Fuks, FeynRules 2.0-A complete toolbox for treelevel phenomenology, Comput. Phys. Commun. 185, 2250 (2014).

[58] C. Degrande, Automatic evaluation of UV and R2 terms for beyond the Standard Model Lagrangians: A proof-ofprinciple, Comput. Phys. Commun. 197, 239 (2015).

[59] T. Hahn, Generating Feynman diagrams and amplitudes with FeynArts 3, Comput. Phys. Commun. 140, 418 (2001).

[60] C. Degrande, C. Duhr, B. Fuks, D. Grellscheid, O. Mattelaer, and T. Reiter, UFO-The Universal FeynRules Output, Comput. Phys. Commun. 183, 1201 (2012).

[61] J. Alwall, R. Frederix, S. Frixione, V. Hirschi, F. Maltoni, O. Mattelaer, H.-S. Shao, T. Stelzer, P. Torrielli, and M. Zaro, The automated computation of tree-level and next-toleading order differential cross sections, and their matching to parton shower simulations, J. High Energy Phys. 07 (2014) 079.

[62] R. D. Ball et al. (NNPDF Collaboration), Parton distributions for the LHC Run II, J. High Energy Phys. 04 (2015) 040 .

[63] A. Buckley, J. Ferrando, S. Lloyd, K. Nordström, B. Page, M. Rüfenacht, M. Schönherr, and G. Watt, LHAPDF6: Parton density access in the LHC precision era, Eur. Phys. J. C 75, 132 (2015).

[64] P. Artoisenet, R. Frederix, O. Mattelaer, and R. Rietkerk, Automatic spin-entangled decays of heavy resonances in Monte Carlo simulations, J. High Energy Phys. 03 (2013) 015 .

[65] J. Alwall, C. Duhr, B. Fuks, O. Mattelaer, D. G. Öztürk, and C.-H. Shen, Computing decay rates for new physics theories with FeynRules and MadGraph 5_aMC@NLO, Comput. Phys. Commun. 197, 312 (2015).

[66] S. Frixione and B. R. Webber, Matching NLO QCD computations and parton shower simulations, J. High Energy Phys. 06 (2002) 029.

[67] J. de Favereau, C. Delaere, P. Demin, A. Giammanco, V. Lemaître, A. Mertens, and M. Selvaggi (DELPHES 3 Collaboration), DELPHES 3, a modular framework for fast simulation of a generic collider experiment, J. High Energy Phys. 02 (2014) 057.

[68] M. Cacciari, G. P. Salam, and G. Soyez, The anti- $k_{t}$ jet clustering algorithm, J. High Energy Phys. 04 (2008) 063. 
[69] M. Cacciari, G. P. Salam, and G. Soyez, FastJet user manual, Eur. Phys. J. C 72, 1896 (2012).

[70] E. Conte, B. Fuks, and G. Serret, MadAnalysis 5, a userfriendly framework for collider phenomenology, Comput. Phys. Commun. 184, 222 (2013).

[71] E. Conte, B. Dumont, B. Fuks, and C. Wymant, Designing and recasting LHC analyses with MadAnalysis 5, Eur. Phys. J. C 74, 3103 (2014).

[72] B. Dumont, B. Fuks, S. Kraml, S. Bein, G. Chalons, E. Conte, S. Kulkarni, D. Sengupta, and C. Wymant, Toward a public analysis database for LHC new physics searches using MADANALYSIS 5, Eur. Phys. J. C 75, 56 (2015).

[73] A. L. Read, Presentation of search results: The $C L_{s}$ technique, J. Phys. G 28, 2693 (2002).

[74] C. G. Lester and D. J. Summers, Measuring masses of semiinvisibly decaying particles pair produced at hadron colliders, Phys. Lett. B 463, 99 (1999).

[75] H.-C. Cheng and Z. Han, Minimal kinematic constraints and $m_{T 2}$, J. High Energy Phys. 12 (2008) 063.

[76] CMS Collaboration, Search for supersymmetry in events with at least one soft lepton, low jet multiplicity, and missing transverse momentum in proton-proton collisions at $\sqrt{s}=13 \mathrm{TeV}$, Report No. CMS-PAS-SUS-16-052.

[77] V. Khachatryan et al. (CMS Collaboration), Search for the production of dark matter in association with top-quark pairs in the single-lepton final state in proton-proton collisions at $\sqrt{s}=8 \mathrm{TeV}$, J. High Energy Phys. 06 (2015) 121.

[78] C. Arina et al., A comprehensive approach to dark matter studies: Exploration of simplified top-philic models, J. High Energy Phys. 11 (2016) 111.

[79] B. Fuks and A. Martini, MadAnalysis5 implementation of the CMS search for dark matter production with top quark pairs in the single lepton channel, Report No. CMS-B2G14-004.

[80] Y. Bai, H.-C. Cheng, J. Gallicchio, and J. Gu, Stop the top background of the stop search, J. High Energy Phys. 07 (2012) 110.
[81] M. Aaboud et al. (ATLAS Collaboration), Search for squarks and gluinos in final states with jets and missing transverse momentum at $\sqrt{s}=13 \mathrm{TeV}$ with the ATLAS detector, Eur. Phys. J. C 76, 392 (2016).

[82] S. Banerjee, D. Barducci, G. Bélanger, B. Fuks, A. Goudelis, and B. Zaldivar, Cornering pseudoscalarmediated dark matter with the LHC and cosmology, J. High Energy Phys. 07 (2017) 080.

[83] D. Sengupta, Madanalysis5 implementation of the ATLAS monojet and missing transverse momentum search documented, Phys. Rev. D 94, 032005 (2016).

[84] B. Fuks, S. Banerjee, and B. Zaldivar, MadAnalysis5 implementation of the multijet analysis of ATLAS, Eur. Phys. J. C 76, 392 (2016).

[85] A. Ibarra, A. Pierce, N. R. Shah, and S. Vogl, Anatomy of coannihilation with a scalar top partner, Phys. Rev. D 91 , 095018 (2015).

[86] K. Griest and D. Seckel, Three exceptions in the calculation of relic abundances, Phys. Rev. D 43, 3191 (1991).

[87] A. De Simone, G. F. Giudice, and A. Strumia, Benchmarks for dark matter searches at the LHC, J. High Energy Phys. 06 (2014) 081.

[88] S. El Hedri, A. Kaminska, M. de Vries, and J. Zurita, Simplified phenomenology for colored dark sectors, J. High Energy Phys. 04 (2017) 118.

[89] A. Pierce, N. R. Shah, and S. Vogl, Stop co-annihilation in the minimal supersymmetric Standard Model revisited, Phys. Rev. D 97, 023008 (2018).

[90] A. Mitridate, M. Redi, J. Smirnov, and A. Strumia, Cosmological implications of dark matter bound states, J. Cosmol. Astropart. Phys. 05 (2017) 006.

[91] S. Biondini and M. Laine, Thermal dark matter coannihilating with a strongly interacting scalar, J. High Energy Phys. 04 (2018) 072.

[92] L. Bergstrom and P. Ullio, Full one loop calculation of neutralino annihilation into two photons, Nucl. Phys. B504, 27 (1997). 